\title{
SPECTRALLY TUNABLE LIGHT SOURCE BASED ON A DIGITAL MICROMIRROR DEVICE FOR RETINAL IMAGE CONTRAST ENHANCEMENT
}

\author{
P. Bartczak ${ }^{a}$, D. Čerāne ${ }^{b}$, P. Fältt ${ }^{\text {a }}$ P. Ylitepsa ${ }^{c}$, E. Hietanen ${ }^{c}$, N. Penttinen ${ }^{\text {a }}$, \\ L. Laaksonen ${ }^{\text {d }}$, L. Lensu ${ }^{d}$, M. Hauta-Kasari ${ }^{a}$, and H. Uusitalo ${ }^{c, e}$ \\ a School of Computing, University of Eastern Finland, Yliopistokatu 2, P. O. Box 111, FI-80101, Joensuu, Finland \\ ${ }^{\mathrm{b}}$ Department of Optometry and Vision Science, University of Latvia, 8 Kengaraga Street, Riga, LV-1063, Latvia \\ ${ }^{c}$ Department of Ophthalmology, University of Tampere, Kalevantie 4, FI-33014 University of Tampere, Tampere, Finland \\ ' Machine Vision and Pattern Recognition Laboratory, Lappeenranta University of Technology, \\ P.O. Box 20, Skinnarilankatu 34, FI-53851 Lappeenranta, Finland \\ e SILK Research and Development Center for Ophthalmic Innovations, School of Medicine, \\ Biokatu 14, FI-33014 University of Tampere, Tampere, Finland \\ E-mail: piotr.bartczak@uef.fi
}

Received 21 May 2015; revised 29 June 2015; accepted 29 September 2015

\begin{abstract}
Different optical methods for retinal imaging provide a significant improvement for image analysis and help with data interpretation. The use of tunable light sources, which have been optimized for contrast enhancement of various retinal features or lesions in retinal images, could simplify the eye fundus examination through enhanced image quality. In this study, we have developed and described a research prototype which consists of a spectrally tunable light source based on a digital micromirror device which is further coupled to a fundus camera. The overall aim of this construction was to generate illuminations optimized for enhanced retinal image feature visibility. The optimized illumination conditions were compared to traditional red-free imaging and the measurements were executed for an artificial eye followed by in vivo measurements of the eyes of three volunteers. In all cases, the retinal image contrast was observed to improve compared to the traditional red-free imaging. Depending on the observed retinal feature, the perceptual improvements in the contrast varied from a few percent to nearly 70 percent.
\end{abstract}

Keywords: biomedical optical imaging, diabetes, lighting control, tunable light source, micromirrors

PACS: 87.57.-s, 87.85.Ox, 87.57.cj

\section{Introduction}

The bottom of the human eye, i. e. ocular fundus, can provide valuable information about the medical condition of a person. In addition to eye diseases like glaucoma, age-related macular degeneration and retinitis pigmentosa, other medical conditions such as intracranial hypertension as well as blood and circulationrelated conditions like diabetes mellitus can also cause visible changes in the visual appearance of the ocular fundus. In hospitals and eye clinics, fundus photography is the standard method for observing and recording the condition of the retina. Here, photographs are captured from the bottom of the eye through a microscope system, also known as a fundus camera. Typically, broadband light sources which contain all the wavelengths over the visible range of light $(400-700 \mathrm{~nm})$ are used for imaging, as they produce realistic colors in the fundus images. Often, a broad bandpass filter which transmits mostly green light (a "red-free" filter) is used to take monochromatic images of the retina. In red-free images, blood-related features are emphasized, which allows for easier detection of vascular abnormalities. However, the use of broadband illumination such as white or red-free light is not necessarily optimal for the detection of clinically relevant features from retinal images. Approaches like multispectral imaging, the use of optical narrow bandpass filters, or the use of illuminations with optimized spectral distributions can significantly increase the visibility and visual contrast of interesting features and landmarks in retinal images.

A reduction of visible features in longer wavelengths was observed in [1], where ocular fundus was 
spectrally imaged with several light emitting diodes (LEDs) as a light source. Another type of system allowing extraction of histological parameters from multispectral images was based on a liquid crystal tunable filter, which could provide a larger number of spectral colour channels between 400-700 nm [2].

Different approach to multispectral imaging of the fundus is snapshot imaging, where the fundus is illuminated with broadband white light, and the reflected light is separated into its spectral components by a diffractive optical element. This results in a simultaneous capture of several spectral channel images. Snapshot spectral imaging has been used for obtaining spectral signatures of oxy-hemoglobin and macular pigments [3]. Francis et al. used a commercial spectrally tunable light source for capturing several images under selected wavelengths which resulted in highlighting in different layers of the posterior segments [ $[$. Another application of multispectral retinal data is the use of selected filters at six specified wavelengths. The distribution maps of the main pigments and retinal hemoglobins in the fundus were derived from the acquired multispectral images [5].

In addition to the applications focused on studying the ocular media, retina's oxygen saturation and retina's pigment content, multispectral imaging has also been applied in the case of diabetic retinopathy. Images acquired through 30 narrow bandpass interference filters show significant visibility enhancement of diabetic lesions compared with conventional RGB images [6].

Furthermore, gathered spectral data has been used to calculate optimized illuminations for the visual detection of retinal lesions [7]. From a set of 72 spectral fundus images, diabetic lesion/surrounding healthy tissue spectrum pairs were manually selected. The authors then used the particle swarm optimization algorithm in order to obtain the spectral shapes of the optimal illuminations for different types of lesions. Each obtained illumination spectrum corresponds to the optimal solution which maximizes the contrast between a selected lesion type and the healthy background. Computational example images presented in this study showed that the contrast and visibility of the retinal features could be improved even without additional image post-processing.

In this paper, the applicability of these spectrally optimized illuminants for retinal imaging is studied. a spectrally tunable light source based on a digital micromirror device (DMD) was constructed in order to produce illuminations of desired spectral shapes [8]. This DMD light source was connected to a commercial fundus camera and fundus images were captured from the eyes of two diabetic and one non-diabetic volunteers.

\section{Methodology}

The prototype device for retinal image contrast enhancement was built by coupling a self-designed DMD-based spectrally tunable light source (Discovery 1100, Texas Instruments, Inc., USA) and a Retiga4000DC monochrome digital charge-coupled device (CCD) camera (QImaging, Canada) to a commercial Topcon TRC-50V (Topcon Corporation, Japan) fundus camera (Fig. 1). However, a number of modifications were done before integrating all components. First, all unnecessary in-built elements, such as the halogen illumination bulb and the xenon flash tube, were removed from the interior optical path of the retinal camera. Next, the original $35 \mathrm{~mm}$ photographic film camera, relay lens, and controlling electronics were unmounted from the Topcon's upper-port and the exposed lockring was covered by a plug to protect optics from dust and stray light.

The spectrally tunable light source used in this study consists of a high-power broadband light source (Thorlabs HPLS-30-04, Thorlabs Inc., USA), followed by a slit, and a diffraction grating. The dispersed spectrum is focused onto the DMD's micromirrors (Fig.2).

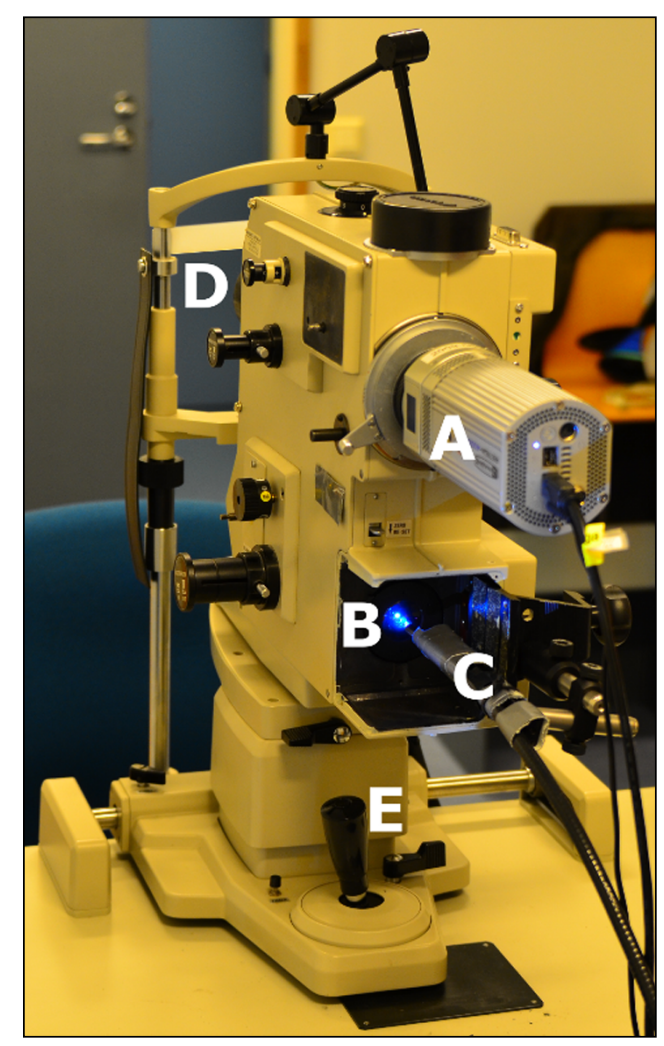

Fig. 1. The modified fundus camera used for retinal imaging: A is Retiga-4000DC monochrome CCD camera, $B$ is illumination collection optics, $C$ is liquid light guide, $\mathrm{D}$ is forehead and chin rest, $\mathrm{E}$ is omni-directional joystick control. 


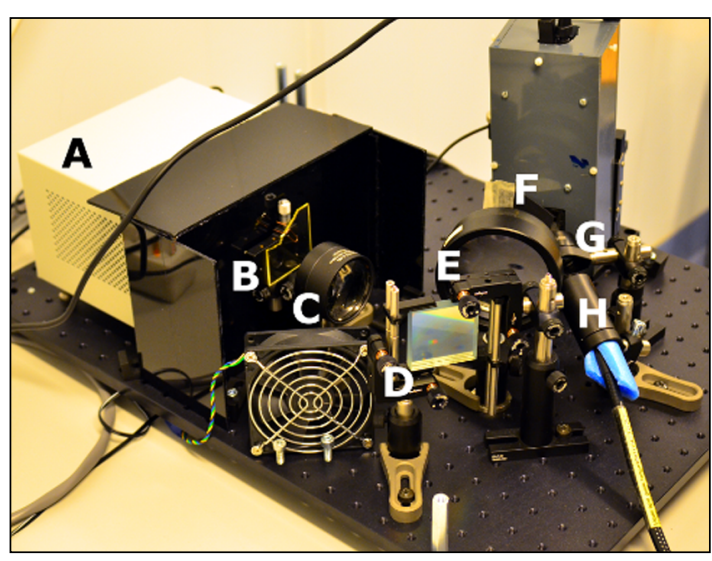

Fig. 2. The DMD-based spectrally tunable light source used in this study: A is light source, B is entrance slit with a UV filter, $\mathrm{C}$ is collimating lens, $\mathrm{D}$ is diffraction grating, $\mathrm{E}$ are focusing lenses, $\mathrm{F}$ is DMD, $\mathrm{G}$ is focusing lens, $\mathrm{H}$ is liquid light guide.

The DMD is mounted onto an articulating base which can be adjusted manually providing free spherical motion over a complete hemisphere. The micromirrors are arranged in a two-dimensional $1024 \times 768$ grid. The size of each micromirror is $13.68 \times 13.68 \mu \mathrm{m}^{2}$ and the total width and height of the mirror array are approximately 14.0 and $10.5 \mu \mathrm{m}$, respectively. The inclination of each micromirror can be switched between two modes: "on" and "off". In the deactivated mode, the reflected light is directed toward a light trap and therefore selected wavelength components are vanished, whereas in the activated state of a micromirror, the wavelength components are reflected and focused into a liquid light guide. In other words, desired spectral power distribution (SPD) is obtained by switching "on" or "off" the appropriate micromirrors. The liquid light guide was attached to the fundus camera's illumination collection optics. The optical setup describing the light propagation path and DMD working principle is presented in Fig. 3.

The whole micromirror array can be controlled by loading into the DMD's memory a monochromatic $1024 \times 768$ bitmap image file (BMP), where the columns are used for wavelength selection and the amount of activated row pixels in each column determines wavelength's intensity. A dedicated software for reading and displaying a BMP file (later referred to as a pattern) and a pattern generation algorithm were implemented for the purpose of this study. A desired number of generated patterns can be loaded into the DMD's buffer and then displayed separately or in a sequential mode.

The CCD camera is cooled in order to reduce dark noise and thermal drift. The CCD detector array size is $2048 \times 2048$ pixels with a dynamic range of 12 bits. The camera was controlled by the QCapture Pro 6.0 software provided by the manufacturer. The camera was operated in two modes: a live preview mode for real time monitoring, and a sequential mode for fast image acquisition.

The retinal imaging procedure was as follows: The DMD-based spectrally tunable light source was allowed to stabilize for at least 20 minutes before the beginning of the image acquisition. The live preview mode was used for positioning and focusing of the fundus camera. Micromirror patterns corresponding to the optimal illuminants were loaded into the DMD's buffer one by one and images were taken of the subject's fundus for each illuminant separately.

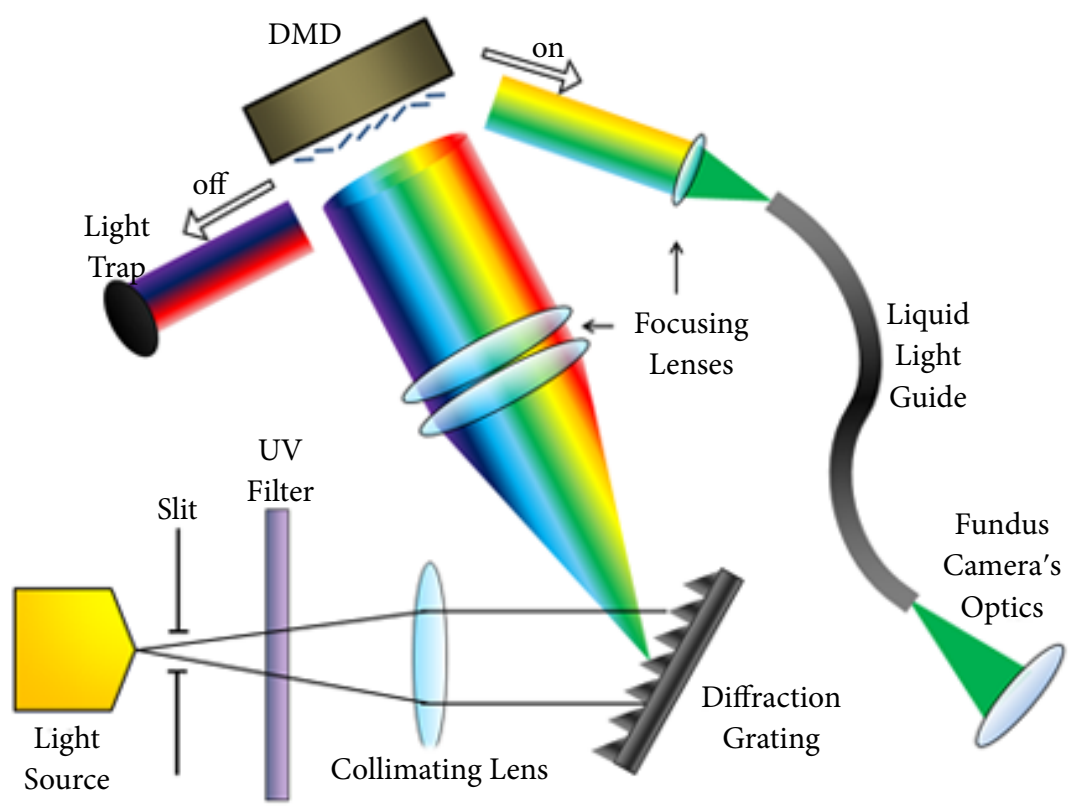

Fig. 3. Optical setup schematics of the designed DMD-based spectrally tunable light source. 
The camera parameters: gain, offset, gamma, dynamic range, and binning were set to $6,0,1,8$-bit, and $2 \times 2$, respectively. The gain-value and binning were selected to shorten the exposure time, while the bit depth setting was changed for easier image previewing. The camera was programmed to capture 10 images, as quickly as possible, and to automatically save all images to the PC's hard drive. Image acquisition produced 8 -bit grayscale TIFF-images $(1024 \times 1024$ pixels $)$. After capturing a whole set of 10 images the camera operator investigated the images. In the case of an unfocused or a blurred set of images the imaging was repeated until satisfactory results were obtained. Because of the constant involuntary movements of the eye, an external fixation target was used. The exposure times varied between $0.1-0.3 \mathrm{~s}$.
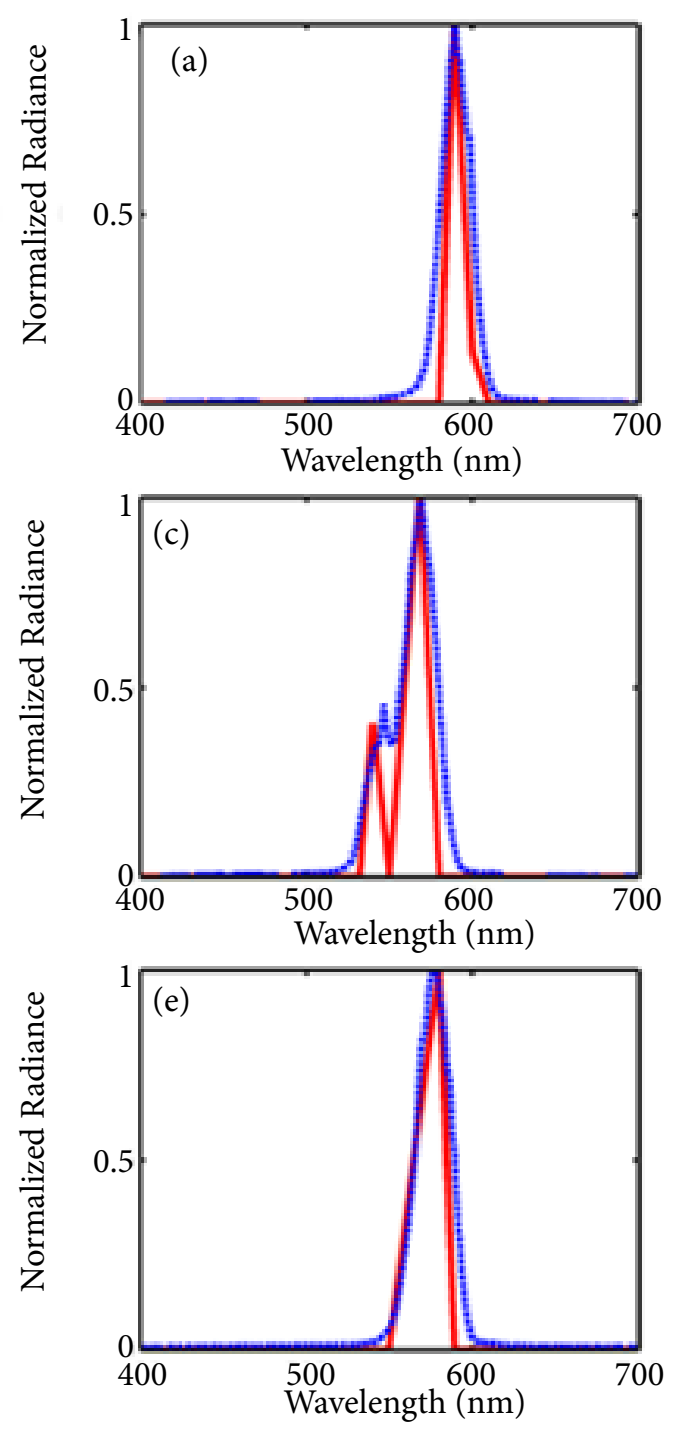

Before imaging, the volunteers' pupils were dilated with a mydriatic (Oftan Tropicamid $5 \mathrm{mg} / \mathrm{ml}$, Santen $O y$, Finland). Forehead and chin rests were used for subject's head stabilization.

Computationally determined optimal spectral power distributions of illuminations which maximize the contrast between various retinal features and the surrounding healthy tissue were described in the previous study 伂. The DMD-based spectrally tunable system was used for implementation of optimized illuminations for the following cases: (a) maximum contrast between arteries and veins, (b) blot bleedings, (c) hard exudates, (d) macula, and (e) microaneurysms (Fig. A). The spectra obtained from the DMD-based spectrally tunable light source (blue dashed lines) differ from the calculated optimized SPDs (solid red
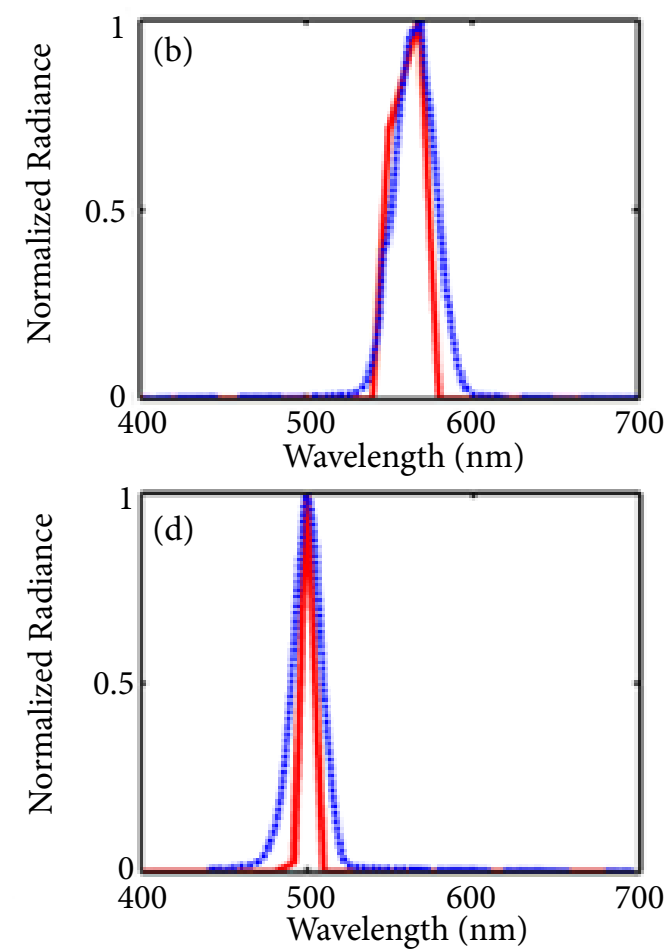

Fig. 4. The spectral power distributions of the optimal illuminants for contrast enhancement of (a) arteries vs veins, (b) blot bleedings, (c) hard exudates, (d) macula, (e) microaneurysms. The solid and dashed lines correspond to calculated and implemented spectral power distributions, respectively. In (c), the small spike at $545 \mathrm{~nm}$ is caused by the spikiness of the light source. 
lines). The spectrum produced by the DMD light source is typically broader than the target spectrum. This is due to the fact that in order to get accurate control of the light's spectral shape, each micromirror column should correspond to as narrow spectral band as possible. However, as the width of each column is only $13.68 \mu \mathrm{m}$, achieving the optimal positioning of the DMD can be very challenging. Furthermore, obtaining the optimal case where each mirror corresponds to a really narrow spectral band was constrained by the application selected in this study. Too low optical power of the light entering the eye would result in long exposure times of the camera. This combined with involuntary eye movements would result in blurry and dark images. Therefore, the width of the slit located after the light source was set so that there was enough light for imaging while maintaining reasonable spectral resolution. Using a more powerful light source and decreasing the slit's width would make it possible to create more accurate spectral shapes.

The maximum irradiance at the plane of the eye's cornea was $20 \mathrm{~mW} / \mathrm{cm}^{2}$ (white light, full power, all DMD-mirrors "on"). With the maximum power, the ANSI safety standard permits 12 minutes of continuous exposure to the retina [9]. For all the implemented illumination spectra, the optical irradiances were well below the maximum value, therefore allowing safe continuous exposures of several tens of minutes. During the actual imaging, only exposures of few seconds were needed.

The study was approved by the Research Ethics Committee of the Pirkanmaa Hospital District (Finland) and the trials followed the ethical tenets of the Declaration of Helsinki. An informed consent was acquired from the volunteers before the trials. The imaging was done at the Tampere University Hospital (Tampere, Finland).

A total of three voluntary human subjects participated in the fundus imaging test. These included two diabetic patients and one healthy control subject. For all cases both eyes were imaged.

\section{Results and discussion}

Initial tests of the modified fundus imaging system were conducted for the system performance characterization purpose. Preliminary measurements were carried out using an artificial eye (Carl Zeiss Meditec, Germany). Resulting images were normalized to the unit exposure time (Fig. 5).

For all cases a donut-shaped artifact is highly noticeable (see arrow at Fig. 5(b)). It is a common phenomenon caused by an annular illumination pattern used by the fundus camera obtained by placing a cir-
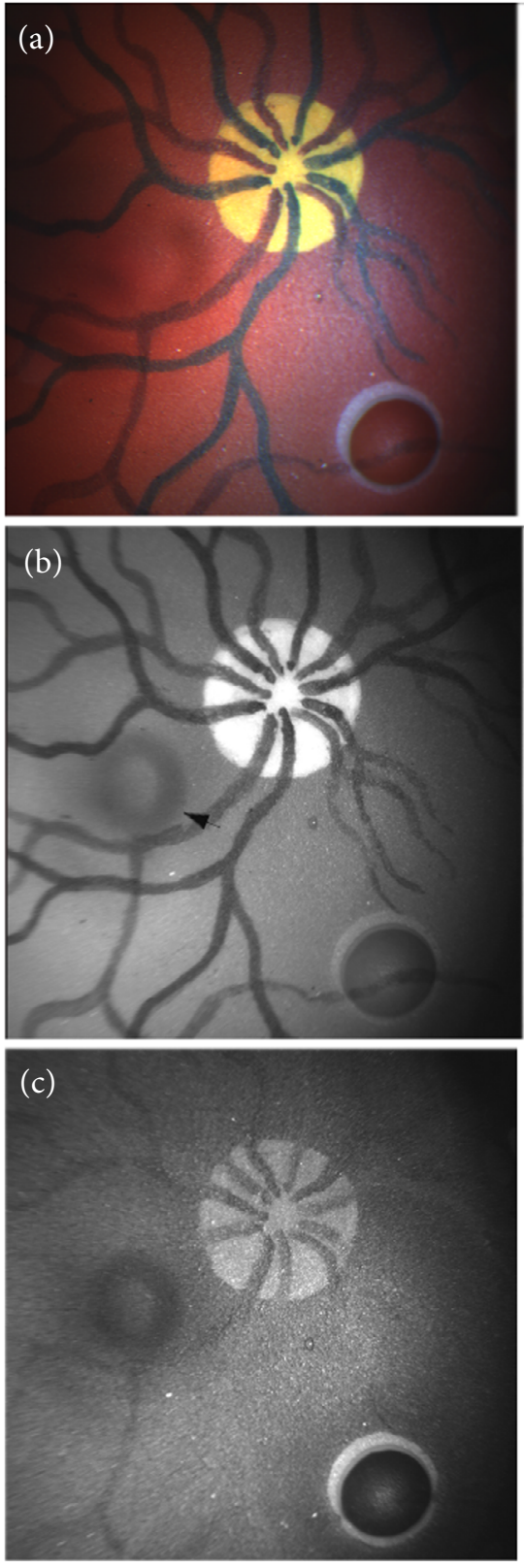

Fig. 5. Images from an artificial eye showing: (a) RGB color image, (b) optimal illumination for veins vs arteries, (c) optimal illumination for the macula. The dark circle in the images is caused by the fundus camera optics.

cular obstruction in the microscope's optical path used to reduce artifacts caused by corneal reflections. This configuration allows faster focusing and more uniform illumination onto the corneal surface on the eye [10]. In addition, a common problem, known as vignetting, is noticeable for each acquired image. The central area of the artificial eye is brighter than the image corners. The image areas corrupted by described illumination imperfections were not examined. Furthermore, uneven illumination correction through Gaussian smoothing was applied for every studied image in the following part of the experiment [11]. 
Figure 6 shows the comparative visual performance of fundus images from the retinas of three voluntary human subjects. The image region containing a retinal feature, for which an optimal illuminant was designed (right column), was cropped and compared with the same region of the retina acquired under red free illumination (left column). Each pair of arrows specifies retinal landmarks (Fig. $6(\mathrm{a})$, (c)) or different lesions of diabetic retinopathy (Fig. 6(b), (d), (e)).

From the locations of the arrows, $3 \times 3$ pixel areas were selected and a mean value was calculated for

(a)

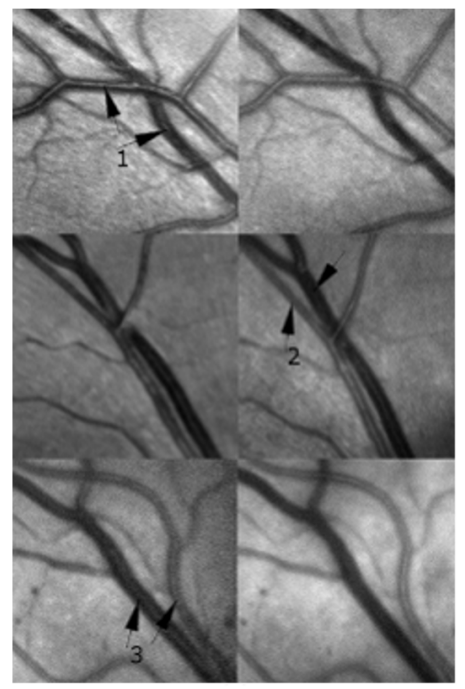

(c)

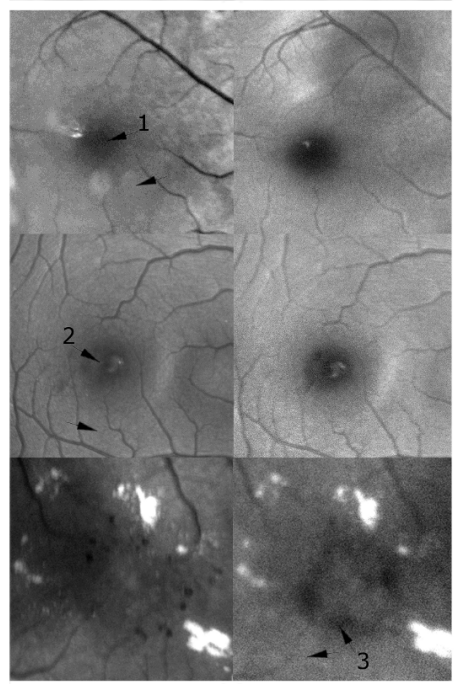

(e)

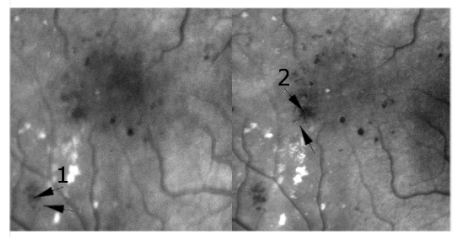

each area. Then a Michelson contrast between a pair of grayscale values was calculated as follows [12]:

$$
C_{\mathrm{M}}=\frac{I_{\max }-I_{\min }}{I_{\max }+I_{\min }}
$$

where $I_{\max }$ and $I_{\min }$ are the larger and the smaller of the values, respectively. The Michelson contrast values are shown in the Table, where it can be seen that in almost all cases the contrast improvement reaches several tens of percent. Furthermore, in the case of

(b)

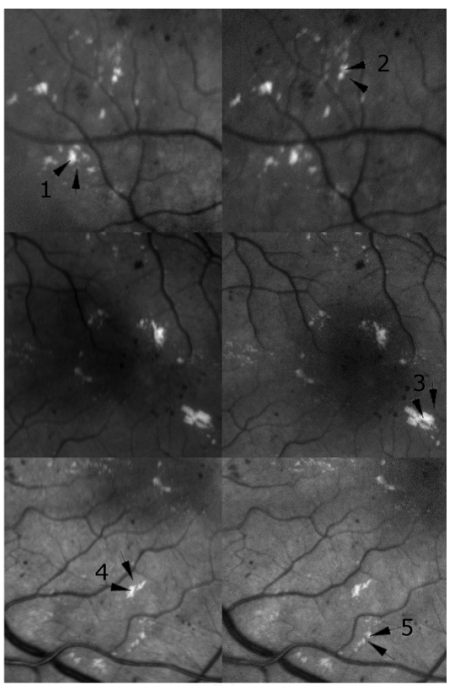

(d)

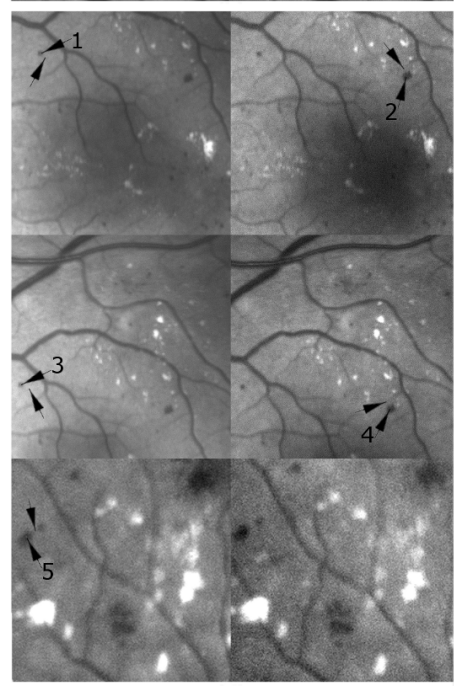

Fig. 6. Example images from the retinal imaging subdivided into the separate blocks for cases: (a) arteries vs veins, (b) hard exudates, (c) macula, (d) microaneurysms, and (e) blot bleedings. In each block, left column shows red-free illumination and right column shows optimal illumination images. Each row corresponds to different regions of interest. Arrows indicate areas from which contrast values were calculated (see Table). Areas are the same for both images in a pair. 
Table. Comparison of contrast between images taken under red-free and optimal illuminants. Also, improvement gained by using an optimal illuminant versus red-free is shown.

\begin{tabular}{|c|c|c|c|c|}
\hline \multirow[b]{2}{*}{ Case } & \multicolumn{4}{|c|}{ Contrast } \\
\hline & $\dot{0}$ & 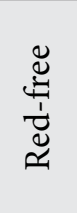 & 胥 & 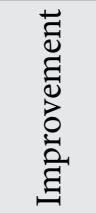 \\
\hline \multirow{3}{*}{$\begin{array}{l}\text { Arteries vs veins } \\
\text { (a) }\end{array}$} & 1 & 0.36 & 0.52 & 30.77 \\
\hline & 2 & 0.14 & 0.46 & 69.57 \\
\hline & 3 & 0.26 & 0.41 & 36.59 \\
\hline \multirow{5}{*}{$\begin{array}{l}\text { Hard exudates } \\
\text { (b) }\end{array}$} & 1 & 0.44 & 0.47 & 6.38 \\
\hline & 2 & 0.44 & 0.47 & 6.38 \\
\hline & 3 & 0.14 & 0.21 & 33.33 \\
\hline & 4 & 0.29 & 0.32 & 9.38 \\
\hline & 5 & 0.27 & 0.32 & 15.63 \\
\hline \multirow{3}{*}{$\begin{array}{l}\text { Macula } \\
\text { (c) }\end{array}$} & 1 & 0.45 & 0.64 & 29.69 \\
\hline & 2 & 0.37 & 0.56 & 33.93 \\
\hline & 3 & 0.15 & 0.47 & 68.09 \\
\hline \multirow{5}{*}{$\begin{array}{c}\text { Microaneurysms } \\
\text { (d) }\end{array}$} & 1 & 0.16 & 0.19 & 15.79 \\
\hline & 2 & 0.19 & 0.33 & 42.42 \\
\hline & 3 & 0.13 & 0.20 & 35 \\
\hline & 4 & 0.20 & 0.32 & 37.5 \\
\hline & 5 & 0.28 & 0.81 & 65.43 \\
\hline \multirow{2}{*}{$\begin{array}{l}\text { Blot bleedings } \\
\text { (e) }\end{array}$} & 1 & 0.26 & 0.38 & 31.58 \\
\hline & 2 & 0.3 & 0.44 & 31.82 \\
\hline
\end{tabular}

hard exudates, where the gained improvement is the lowest, there is still a minimum of six percent of improvement. Hard exudates are relatively strongly reflected in the visible range of light, and this can be noticed by comparing visibility of hard exudates from images acquired for other illuminant cases (Fig. 5 (c), (d), (e)).

Despite of these lower values, the mean value of all of the improvements shown in the Table is 33 percent, which indicates great potential for this method and device.

\section{Conclusions}

A spectrally tunable light source based on a digital micromirror device was constructed and optimal illuminants for retinal image contrast enhancement were implemented. Fundus images from the retinas of three voluntary human subjects were acquired by using a tunable DMD light source and a modified fundus camera. Acquired images were compared against red-free images which are typically used in health care systems for the inspection and documentation of the ocular fundus. Depending on the ob- served retinal lesions (e. g. hard exudates) or other features (e. g. macula) the perceptual improvements in the contrast varied from a few percent to nearly 70 percent. The results are promising as the studied spectrally optimized illuminants could be potentially incorporated into existing retinal imaging systems as well as could improve the future retinal imaging systems used for medical diagnosis.

\section{Acknowledgements}

The authors would like to thank the Academy of Finland for funding (ReVision project, Funding Decision No. 259530). The strategic funding from the Faculty of Science and Forestry, University of Eastern Finland, is also acknowledged. The authors would like to thank Pauli Ahonen (Ahosen Musiikki Oy, Tampere) for support and assistance with the imaging.

\section{References}

[1] N.L. Everdell, I.B. Styles, A. Calcagni, J. Gibson, J. Hebden, and E. Claridge, Multispectral imaging of the ocular fundus using light emitting diode illumination, Rev. Sci. Instrum. 81, 093706-1-9 (2010), http://dx.doi.org/10.1063/1.3478001

[2] I.B. Styles, A. Calcagni, E. Claridge, F. OrihuelaEspina, and J.M. Gibson, Quantitative analysis of multispectral fundus images, Med. Image Anal. 10, 578-597 (2006), http://dx.doi.org/10.1016/j. media.2006.05.007

[3] G. Liang, R.T. Smith, and T.S. Tkaczyk, Snapshot hyperspectral retinal camera with the Image Mapping Spectrometer (IMS), Biomed. Opt. Express 3(1), 4854 (2012), http://dx.doi.org/10.1364/boe.3.000048

[4] R.P. Francis, K.J. Zuzak, and R. Ufret-Vincenty, Hyperspectral retinal imaging with a spectrally tunable light source, Proc. SPIE 7932, 793206 (2011), http://dx.doi.org/10.1117/12.873891

[5] A. Calcagni, J.M. Gibson, I.B. Styles, E. Claridge, and F. Orihuela-Espina, Multispectral retinal image analysis: a novel non-invasive tool for retinal imaging, Eye 25(12), 1562-1569 (2011), http://dx.doi. org/10.1038/eye.2011.202

[6] P. Fält, J. Hiltunen, M. Hauta-Kasari, I. Sorri, V. Kalesnykiene, and $\mathrm{H}$. Uusitalo, in: Proceedings of the 16th Scandinavian Conference on Image Analysis, eds. A.-B. Salberg, J.Y. Hardeberg, and R. Jenssen (Springer-Verlag Berlin, Heidelberg, 2009) pp. 149158, http://dx.doi.org/10.1007/978-3-642-022302_16

[7] P. Fält, J. Hiltunen, M. Hauta-Kasari, I. Sorri, V. Kalesnykiene, J. Pietilä, and H. Uusitalo, Spectral imaging of the human retina and computationally determined optimal illuminants for diabetic retinopathy lesion detection, J. Imaging Sci. 
Technol. 55, 030509-1-10 (2011), http://dx.doi. org/10.2352/j.imagingsci.technol.2011.55.3.030509

[8] S. Tominaga, T. Horiuchi, H. Kakinuma, and A. Kimachi, in: 17th Color and Imaging Conference Final Program and Proceedings (Society for Imaging Science and Technology, 2009) pp. 133138, http://www.ingentaconnect.com/content/ist/ cic/2009/00002009/00000001

[9] F.C. Delori, R.H. Webb, and D.H. Sliney, Maximum permissible exposures for ocular safety (ANSI 2000), with emphasis on ophthalmic devices, J. Opt. Soc. Am. A 24, 1250-1265 (2007), http:// dx.doi.org/10.1364/josaa.24.001250
[10]K. Tran, T.A. Mendel, K.L. Holbrook, and P.A. Yates, Construction of an inexpensive, handheld fundus camera through modification of a consumer "point-and-shoot" camera, Invest. Ophthalmol. Vis. Sci. 53(12), 7600-7607 (2012), http://dx.doi.org/10.1167/iovs.12-10449

[11]F.W. Leong, M. Brady, and J.O.D. McGee, Correction of uneven illumination (vignetting) in digital microscopy images, J. Clin. Pathol. 56(8), 619-621 (2003), http://dx.doi.org/10.1136/ jcp.56.8.619

[12] A.A. Michelson, Studies in Optics (University of Chicago Press, Chicago, USA, 1927).

\title{
SPEKTRIŠKAI DERINAMAS ŠVIESOS ŠALTINIS TINKLAINĖS VAIZDO KONTRASTUI DIDINTI, NAUDOJANTIS SKAITMENINI MIKROVEIDRODI
}

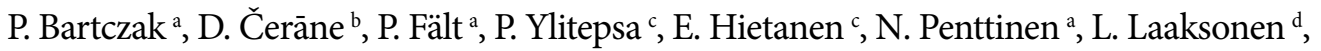 \\ L. Lensu ${ }^{\mathrm{d}}$, M. Hauta-Kasari ${ }^{\mathrm{a}}, \mathrm{H}$. Uusitalo ${ }^{\text {c,e }}$ \\ ${ }^{\text {a }}$ Rytu Suomijos universitetas, Joensuu, Suomija \\ ${ }^{\mathrm{b}}$ Latvijos universitetas, Riga, Latvija \\ ${ }^{c}$ Tamperés universiteto Oftalmologijos katedra, Tamperè, Suomija \\ ${ }^{\mathrm{d}}$ Lappeenranta technologijos universitetas, Lappeenranta, Suomija \\ ${ }^{\mathrm{e}}$ Tamperés universiteto Medicinos mokykla, Tamperé, Suomija
}

